\title{
Annotating Narrative Levels: Review of Guideline No. 6
}

\author{
Natalie M. Houston
}

01.15 .20

Article DOI: $10.22148 / 001 c .11774$

Journal ISSN: 2371-4549

Cite: Natalie M. Houston, "Annotating Narrative Levels: Review of Guideline No. 6," Journal of Cultural Analytics. January 15, 2020. doi: 10.22148/001c.11774

The framing of Guideline VI within the pedagogical situation of a class on "Digital Methods in Literary Studies" is helpful in pointing out some of the ways in which the theory and practice of annotation can serve students of literature, as well as eventually contributing to computational analysis. Above all, annotation necessitates firm decisions, as the authors describe: "Rather than let an ambiguous text stay ambiguous, they simply had to decide for one option in order to be able to annotate a passage and had to justify their choice with reference to the whole text or to adapt the guidelines in order to address and document the ambiguity" (3). This remark highlights the challenge in developing annotation guidelines so that they can be used consistently by different communities of users without modifications. The authors note several points of debate within the class that are relevant to the overall shared task and its evaluation: the feasibility of developing annotation guidelines that could be applied to a wide range of literary texts; the involved levels of textual interpretation that some kinds of annotation require; and the effect of prior study or knowledge on an annotator's ability to discern or interpret narrative levels. As the shared task proceeds, it may be necessary to specify the applicability of the annotation guidelines to works from particular genres, time periods, or languages.

This guideline usefully deploys key concepts from narratological theory, including Genette's outline of narrative levels and types of narrators, Ryan's focus on the illocutionary and ontological boundaries, and Nelles's notion of the narratee. 
Translating these theories into an outline of annotation choices is useful. But the textual examples that are provided are not sufficiently explained, and so do not adequately serve to guide a user's potential application of these categories to another text. Although Shelley's Frankenstein is a well-known novel, it presents several levels of narratological complexity that ought to be better explained if it is to be used as an exemplar. To readers who have not spent weeks immersed in the task of annotating Frankenstein, many of these examples of level change or narrator change will not be clear.

In particular, the following aspects of this Guideline could be revised for greater clarity and applicability:

(1) The authors state that they decided to annotate "the point between two different narrative levels," yet the examples show the use of paired tags around sections of the narrative, which seems to contradict this statement.

(2) Some of the examples are drawn from Frankenstein, and others appear to be invented examples. The sources and selection of example texts should be clearly identified and explained.

(3) The examples are presented together as a block paragraph that often suggests they are taken sequentially from the novel, when in fact they are not. (i.e., \#3 Narrator Change presents extracts from chapter 11 and then chapter 9). What are the closing tags closing? This formatting error can lead a reader unfamiliar with Shelley's novel to think all this text comes from one sequence in the novel.

(4) Most of the examples taken from Frankenstein are drawn from the opening lines of chapters or letters presented within chapters. No discussion is offered in the Guideline about whether or how particular attention ought to be paid to chapter or section boundaries in the literary text.

This Guideline also raises a theoretical point that ought to be considered in the shared task. The authors raise the question about the mediation of embedded narratives when they discuss whether the Creature should be considered the narrator of its own story, which is doubly mediated by other narrators in the novel Frankenstein. Many nineteenth-century novels, such as The Moonstone and Dracula, deliberately exploit textual mediation by presenting documents or letters that are ostensibly transcribed or read aloud by other characters. Whether such mediations constitute a form of narrative level or not should be considered in the annotation guidelines.

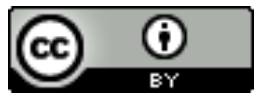


Unless otherwise specified, all work in this journal is licensed under a Creative Commons Attribution 4.0 International License. 